\title{
Should we operate on all patients with COVID-19 and proximal femoral fractures? An analysis of thirty, sixty, and ninety day mortality rates based on patients' clinical presentation and comorbidity: a multicentric study in Northern Italy
}

\author{
Federico Fusini ${ }^{1}$ (D) Alessandro Massè ${ }^{2} \cdot$ Salvatore Risitano $^{3} \cdot$ Andrea Ferrera $^{2} \cdot$ Emilio Enrietti $^{2} \cdot \mathrm{Kristijan} \mathrm{Zoccola}^{4}$. \\ Giuseppe Bianco ${ }^{1} \cdot$ Fabio Zanchini ${ }^{5} \cdot$ Gabriele Colò $^{4}$
}

Received: 6 May 2021 / Accepted: 17 July 2021 / Published online: 16 August 2021

(c) SICOT aisbl 2021

\begin{abstract}
Purpose This study aims to evaluate 30-60-90-day mortality of operated proximal femur fractures (PFFs) suffering from COVID-19 and correlation with patients' clinical presentation and comorbidities.

Methods Between February 1, 2020, and December 31, 2020, patients with COVID-19 infection and surgically treated PFF were included. Patients' demographic characteristics, oxygen $(\mathrm{O} 2)$ therapy, comorbidities, and AO type fracture were collected. Chi-square test or Fisher test and hazard ratio were used to assessing the correlation between mortality rate, patient characteristics, and COVID-19 status. Kaplan-Meyer curve was used to analyze 30-60-90-day mortality. Level of significance was set as $p<0.05$.

Results Fifty-six patients (mean age of $82.7 \pm 8.85$ years) were included. Thirty-day mortality rate was $5 \%$, which increased to $21 \%$ at 60 days and 90 days. Eleven patients died, eight due to AO type A-like and three due to AO type B-like fractures. No significant difference in mortality rate between patients with cardiopulmonary comorbidity or no cardiopulmonary comorbidity was found ( $p=0.67)$; a significant difference in patients with chronic obstructive pulmonary disease (COPD) or history of pulmonary embolism (PE) and patients without COPD was found $(p=0.0021)$. A significant difference between asymptomatic/mild symptomatic COVID-19 status and symptomatic COVID-19 status was found $(p=0.0415)$; a significant difference was found for $\mathrm{O} 2$ therapy with $<4 \mathrm{~L} / \mathrm{min}$ and $\mathrm{O} 2$ therapy $\geq 4 \mathrm{~L} / \mathrm{min}(p=0.0049)$.

Conclusion Thirty-day mortality rate of COVID-19 infection and PFFs does not differ from mortality rate of non-COVID-19 PFFs. However, patients with pre-existing comorbidities and symptomatic COVID-19 infection requiring a high volume of O2 therapy have a higher incidence of 60-90-day mortality when surgically treated.
\end{abstract}

Keywords Coronavirus disease $\cdot$ Fracture $\cdot$ Proximal femur $\cdot$ Hip $\cdot$ Joint replacement $\cdot$ Intramedullary nailing

Federico Fusini

fusinif@ hotmail.com

1 Department of Orthopaedic and Traumatology, Regina Montis Regalis Hospital, ASL CN1, Strada S Rocchetto 99, 12084 Mondovì, Italy

2 Department of Orthopaedic and Traumatology, Orthopaedic and Trauma Centre, Città Della Salute E Della Scienza Di Torino, via Zuretti 29, 10126 Turin, Italy
3 Department of Orthopaedic and Traumatology, Maggiore Hospital of Chieri, ASL TO5, via De Maria 1, 10023 Chieri, Italy

4 Department of Orthopaedics and Traumatology, Regional Center for Joint Arthroplasty, ASO Alessandria, Via Venezia 16, 16121 Alessandria, Italy

5 Clinical Orthopaedics, University of Campania "Luigi Vanvitelli”, via L. de Crecchio 4, 80138 Naples, Italy 


\section{Introduction}

Coronavirus disease 2019 (COVID-19) is a new contagious disease caused by severe acute respiratory syndrome coronavirus 2 (SARS-CoV-2). It started in the Chinese district of Wuhan in late December 2019, and it has subsequently spread shortly nationwide and overseas [1,2]. In January 2020, the World Health Organization declared the outbreak a Public Health Emergency of International Concern and a pandemic in March 2020. As of February 7, 2021, more than 105 million cases have been confirmed, with more than 2.31 million deaths attributed to COVID-19 [3]. On March 8, 2020, the Italian government took steps to contain infections, limiting them first to specific geographical areas and then to the entire nation. The consequent blockade allowed people to move from home only and exclusively for work reasons, health, or the purchase of necessities [4]. During this period, the outpatient activity was suspended and reserved only for urgent and non-postponable visits. A similar situation was present for elective surgery, reserving the operating room only for emergencies.

Proximal femur fractures (PFFs) are a significant burden on the healthcare system, with many emergency room visits per day. Assuming that the vast majority of patients fracture their femur at their homes, the incidence of this type of trauma has not substantially changed from before to after the pandemic. The proximal femur fracture is associated with high mortality up to $36 \%[5,6]$ and, above all, with the patient's disability in the post-operative period, when only 40 to $60 \%$ of patients regain their previous level of independence [7]. In addition, throughout the pandemic period of COVID-19, the correct management of this pathology has undoubtedly represented a critical parameter for assessing the quality of the assistance that a health system must be able to provide $[8,9]$. However, it is questionable whether these types of fractures have led to increased post-operative lethality of patients.

The study aims to evaluate the 30-60-90-day mortality of patients suffering from COVID-19, operated PFFs, and the mortality rate based on the patient's clinical presentation at the time of admission and comorbidity.

\section{Materials and methods}

This is a multicentric retrospective observational cohort study of prospectively collected data. All patients with a positive swab for COVID-19 and surgically treated proximal femur fracture were included in the survey from February 1, 2020, to December 31, 2020. Two first-level trauma centers (Regina Montis Regalis Hospital, Mondovì, Italy, and Martini Hospital, Turin, Italy) and one second-level trauma centre (Santi Antonio e Biagio e Cesare Arrigo Hospital, Alessandria, Italy) in Piedmont participated in the study. No restrictions were applied for the type of surgical treatment. Patients underwent proximal femur reduction and fixation (FRF) with cephalomedullary nail for AO 31 A fractures. In AO 31B or C, total hip replacement (THR) or hemiarthroplasty (HeA) was performed, depending on age and clinical presentation, except for type 31B 1 in young patients where patients underwent fixation with percutaneous cannulated screws (CS). Pediatric patients (younger than 18 years old) and PFFs treated conservatively were excluded, while patients with AO 31A-, 31B-, and 31C-like fractures were included.

Demographic characteristics, AO type of fracture, body mass index (BMI), types of comorbidities, American Society of Anesthesiologists (ASA) score, COVID-19 status, and O2 flow in litres per minute prior intervention were recorded. Types of comorbidities were divided into cardiopulmonary (hypertension, atrial fibrillation, myocardial infarction, coronary disease, heart failure, pulmonary embolism, asthma, chronic obstructive pulmonary disease) and others (diabetes, chronic renal failure, active carcinoma, immunosuppression, depression, dementia, Alzheimer, vasculopaties, myelodysplastic syndromes, dyslipidemia). COVID-19 status was graded as asymptomatic and mild symptomatic (only positive COVID-19 swab, with no symptoms or minor symptoms of infection) or symptomatic (pulmonary involvement, ranging from moderate to severe symptoms) at the time of hospital admission.

Thirty-day, 60-day, and 90-day mortality were set as the study's primary outcome, while the correlation between preoperative health status and 30-day mortality was the secondary outcome. Complications were also reported.

\section{Statistical analysis and ethical standards}

Age, BMI, and ASA score were assessed with KolgomorovSmirnov test of normality for normal distribution $(p=0.357$, $p=0.2077$, and $p<0.00001$, respectively); age and BMI were reported as mean and standard deviation, while ASA score was reported as median and interquartile range (IQR). Main outcomes were reported as rate or percentage, while chi-square test or Fisher exact test was used to assess the correlation between mortality rate and patient characteristics. STATA13 software (StataCorp LLC, College Station, TX, USA) was used to perform statistical analysis. Level of significance was set as $p<0.05$.

Kaplan-Meyer analysis was used to analyze 30-day, 60-day, and 90-day mortality, while the hazard ratio was used to compare mortality risk with patients' characteristics. 
The study was conducted according to the Declaration of Helsinki principles and later amendments. Written informed consent from patients or closest family members was obtained before publication. Ethical approval was obtained from the local ethical committee with registration number "COV 23.2020 bis" and further amendments.

\section{Results}

Main demographic characteristics, comorbidities, and COVID-19 status before surgery are reported in Tables 1 and 2 .

Thirty-day survival rate was $95 \%$ (53/56) while the mortality rate was 5\% (3/56); survival rate decreased to $79 \%$ $(45 / 56)$ while mortality rate increases to $21 \%(11 / 56)$ at 60 days and it remains stable until 90 days (Fig. 1). The main drop in survival rate analysis was recorded between 39 and 50 days from surgery (6/56 patients, $10.7 \%$ ), with the climax between 39 and 41 days after surgery (4/56 patients, $7.1 \%$ ). The second month from surgery recorded the highest incidence of death $(8 / 56,14.3 \%)$.
The main demographic characteristics of deceased patients are reported in Table 3.

Thirty-four patients underwent FRF, eight underwent THR, thirteen underwent HeA, and only one CS. Mortality rate was $23.53 \%$ (8/34) for cephalomedullary nail, $12.8 \%$ (1/8) for THR, $15.38 \%$ (2/13) for HeA, and $0 \%(0 / 1)$ for cannulated screws. However, no significant difference was found among groups (FRF $p=0.3628$; THR $p=0.5828$; HeA $p=0.6592$; CS $p=1$; reduction and synthesis vs joint replacement $p=0.5079$ ).

Five complications were recorded: two wound dehiscence ( 2 nails), the migration of the distal locking screw (1 nail), and two prosthesis dislocations (1 THR and $1 \mathrm{HeA}$ ). All complications were treated conservatively, except for the distal locking screw, surgically removed under local anaesthesia.

No significant difference in the rate of mortality between patients with cardiopulmonary comorbidity or no cardiopulmonary comorbidity were found $(p=0.67)$; however, a significant difference in patients with chronic obstructive pulmonary disease (COPD) or history of pulmonary embolism (PE) and patients without COPD were found $(p=0.0021)$.
Table 1 Demographic characteristics of patients included in the study, 30-day, 60-day, and 90-day mortality rate, comorbidities, and COVID status

Table 2 Summary of patients divided into asymptomatic or mildly symptomatic and symptomatic. Respiratory rate, $\mathrm{O} 2$ therapy, survival, and mortality rate were reported

\begin{tabular}{lll}
\hline Patients & Male & Female \\
& 18 & 38 \\
\hline Age & $82.7 \pm 8.85$ & \\
BMI & $26.03 \pm 3.09$ & \\
ASA score & $4(3$ to 4$)$ & \\
AO fracture type & 38 type 31A-like, 18 type 31B-like \\
Mortality & Rate & Percentage \\
Within 30 days & $3 / 56$ & $5.36 \%$ \\
Within 60 days & $11 / 56$ & $19.64 \%$ \\
Within 90 days & $11 / 56$ & $19.64 \%$ \\
Patients & Survival at 3 months & Death within 3 months \\
56 & 45 & 11 \\
Comorbidity & Survival at 3 months & Death within 3 months \\
Cardiopulmonary and others & 37 & 10 \\
Without cardiopulmonary comorbidities & 8 & 1 \\
\hline
\end{tabular}

\begin{tabular}{lll}
\hline COVID19 status & $\begin{array}{l}\text { Asymptomatic/mild symptomatic (no. } \\
\text { of patients) }\end{array}$ & $\begin{array}{l}\text { Sympto- } \\
\text { matic (no. of } \\
\text { patients) }\end{array}$ \\
\hline Patients & 36 & 20 \\
Respiratory rate $\leq 20$ breaths/min & 34 & 4 \\
Respiratory rate $>20$ breath/min & 2 & 16 \\
O2 therapy $<4$ L/min & 33 & 8 \\
O2 therapy $>4$ L/min & 3 & 12 \\
Survival at 3 months & 32 & 13 \\
Deceased within 3 months & 4 & 7 \\
\hline
\end{tabular}




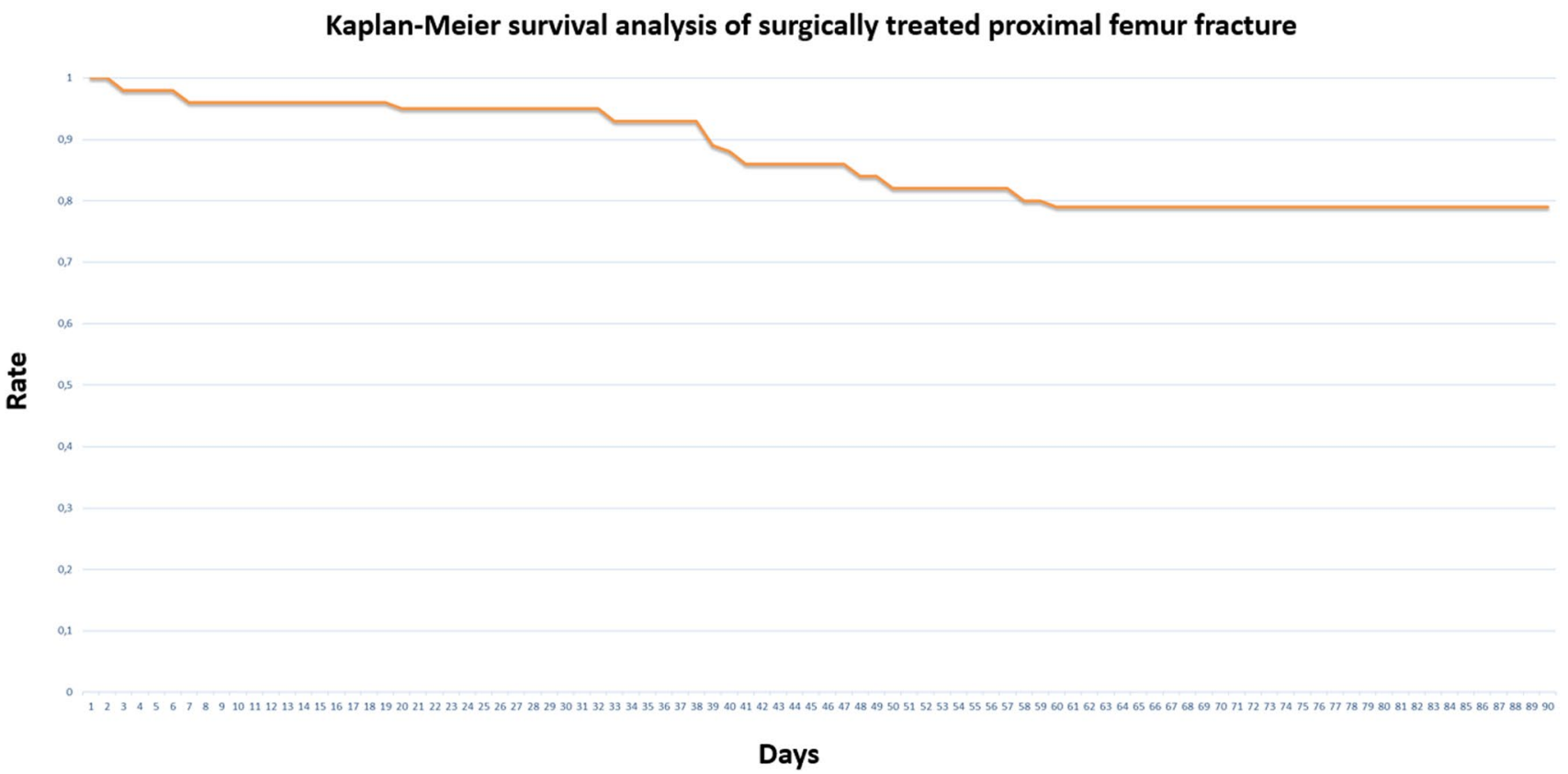

Fig. 1 Kaplan-Meier survivorship curve of patients included in the study

Table 3 Survival rate, statistical analysis, and main characteristics of deceased patients of patients divided by BMI, age, gender, ASA scale, AO type fracture, and $\mathrm{O} 2$ therapy at admission

\begin{tabular}{lll}
\hline & Survival rate & Statistical analysis \\
\hline BMI $<30$ vs BMI $>30$ & $81.63 \%$ vs $71.43 \%$ & $p=0.613$ \\
Age $<75$ vs age $>75$ & $82 \%$ vs $66.67 \%$ & $p=0.5861$ \\
ASA3 vs ASA4 & $87.5 \%$ vs $70.83 \%$ & $p=0.17$ \\
AO type A vs B & $77.14 \%$ vs $85.71 \%$ & $p=0.5079$ \\
Male vs female & $66.67 \%$ vs $86.84 \%$ & $p=0.1463$ \\
\hline & Deceased & Rate \\
\hline Patients & 11 & $100 \%$ \\
Male & 6 & $54.55 \%$ \\
Female & 5 & $45.45 \%$ \\
Mean age (years) & $83.54 \pm 8.15$ & \\
Older than 81 & 8 & $72.73 \%$ \\
Symptomatic & 7 & $63.64 \%$ \\
Cardiopulmonary comorbidity & 10 & $90.91 \%$ \\
Diabetes or carbohydrates intolerance & 5 & $45.45 \%$ \\
AO type 3 & 8 & $72.73 \%$ \\
AO type 4 & 3 & $27.27 \%$ \\
O2 therapy at admission & 10 & $90.91 \%$ \\
O2 flow $>4$ L/min & 7 & $63.64 \%$ \\
\hline
\end{tabular}

Hazard ratio analysis reveals a ratio of 2.25 for cardiopulmonary comorbidity ( $p=0.4263$ ), while the ratio was 8.48 for COPD and PE comorbidities $(p=0.00002)$. Hazard ratio was 4.67 for $\mathrm{O} 2$ therapy $>4 \mathrm{~L} / \mathrm{min}(p=0.0067), 3.85$ for symptomatic patients $(p=0.0205)$ and 0.96 for respiratory rate $>20$ breaths/min $(p=0.9630)$.
A significant difference between asymptomatic/ mild symptomatic and symptomatic patients was found ( $p=0.0415)$; moreover, a significant difference was found for $\mathrm{O} 2$ therapy with $<4 \mathrm{~L} / \mathrm{min}$ and $\mathrm{O} 2$ therapy $\geq 4 \mathrm{~L} / \mathrm{min}$ $(p=0.0049)$. 


\section{Discussion}

The current new pandemic condition led to reconsider some surgical indications treating elderly patients with a proximal femur fracture, questioning about the better management of patients affected by a severe infectious disease which is itself burdened by an elevated risk of life and concerns are also related with the most proper timing of surgery. In this paper, the average post operative mortality rate was $5 \%$ within 30 days and $21 \%$ at 60 days, and it remains stable until 90 days; the cumulative Kaplan-Meier survivorship was $95 \%$ at 30 days, $79 \%$ at two months, and final follow-up.

The mortality rate within 30 days of patients affected by proximal femur fracture and COVID-19 pneumonitis at the same time (5\%) is comparable to values reported in the literature for patients with proximal femur fracture only [10]. Unfortunately, in the present study, the mortality rate increases seriously at 60 days with a cumulative Kaplan-Meier mortality of $21 \%$, which appear quite higher if compared with values reported by other authors before the COVID-19 appearance: after femoral fractures of elderly Italian population at 6-month follow-up, despite a significant increase of deaths, an overall percentage ranging around $15 \%$ was found $[11,12]$.

Since the appearance of COVID-19, the number of patients affected is growing up exponentially worldwide. $143,445,675$ confirmed cases and 3,051,736 deaths are reported as of April 20, 2021, according to the daily situation update by the World Health Organization (WHO) [13]

The percentage of COVID-19-associated death, also expressed as a case-fatality rate (CFR), changes significantly depending on countries, ranging from $0.6 \%$ reported in Iceland to $18.1 \%$ in France [14]. Indeed, differences between countries are also related to demographic characteristics and are well-known as the elderly individuals are the most jeopardized population accounting for the larger portion of fatal cases [15]. About that, Italy is one of the six countries reporting a CFR of $>10 \%$, and approximately $23 \%$ of people aged 65 years old or older may explain this higher CFR [16]. Several influencing factors can also be found on differences in time lags between reporting of infections and deaths: some may be detected on healthcare system organization as infrastructures and surveillance capacities others to patients' general conditions as aged comorbidity [17].

On the other hand, similar considerations can be provided evaluating factors affecting mortality rate after proximal femur fractures. In the literature, the mortality rate of hip fractures in elderly patients is reported to be as high as 20 to $40 \%$ within 1 year after surgery[18], and comorbidity as diabetes, cardiac disease, and COPD or association with surgical delay are recognized as factors predicting an increased mortality risk [11].
In the literature, the potential adverse effect of surgical delay on mortality rate after hip fracture and many authors [19] concluded as an operation within 48 hours was mandatory to achieve higher survivorship. Furthermore, non-surgical treatment was poorly indicated due to association with severe complications and poor clinical outcomes following prolonged immobilization.

An operation within 48 hours is very important for nonCOVID-19 elderly patients, where healthy patients benefit from threefold increased survivorship. However, a 48-hour surgical delay seems to be protective for sicker patients [20]. According to Brogan et al., the global 90-day mortality rate of surgically treated PFF was $14 \%$, and the patients treated after 48 hours did not show increased mortality [21]. According to these results, it is possible to suppose that the higher incidence of mortality in our study could be related to COVID-19 infection. Therefore, we agree with Brogan et al. and Schoeneberg et al. that delay of surgery for treating acute and chronic patient comorbidities optimizes patients' survivorship [21,22].

Dallari et al. reported that few COVID-19-positive patients were surgically treated within 48 hours, and it seems that surgery performed within 48 hours does not associate with lower mortality in this subgroup of patients. However, according to the authors, these data could be influenced by the discrepancy of size between the COVID-19-positive and COVID-19-negative groups [23].

The reason for this discrepancy is certainly multiple and difficult to clarify. To the authors' opinion, in addition to predictors of mortality after surgery for proximal femur fractures already clear in the literature[24], including advanced age, male gender, pre-operative higher ASA grading, and several comorbidities, risk factors directly co-related to the viral infection jeopardizing the general health conditions of the patients might be correctly identified and analyzed.

For patients also affected by COVID-19 infection, the current authors identified the pulmonary function impairment as a severe factor predicting high mortality risk, as in case of previous pulmonary disease (COPD or history of PE) as in terms of clinical presentation of pneumonitis COVID19-related (asymptomatic/mild symptomatic COVID-19 status and symptomatic COVID-19 status $(p=0.0415)$ and difference on $\mathrm{O} 2$ therapy with $<4 \mathrm{~L} / \mathrm{min}$ and $\mathrm{O} 2$ therapy $\geq 4 \mathrm{~L} /$ $\mathrm{min}$ ). These findings are crucial for post-operative care management; they allow to identify patients more likely to need an intensive care unit admission or patients who will benefit from surgery delay. Despite the suggestions of COVIDSurg and GlobalSurg Collaborative, who suggested waiting until seven weeks from COVID-19 infection or resolution of symptoms before undertaking surgery, we strongly believe that PFF surgery cannot be delayed that much. The study was designed for elective surgery; however, delaying surgery for deferrable emergency intervention until improvement of 
symptoms and respiratory function $(\mathrm{O} 2$ therapy with $<4 \mathrm{~L} /$ min) could be a wise compromise [25].

Our research is the first largest and multicentric study considering patients with pandemic COVID-19 infection and surgically treated proximal femur fracture. Most of the data reported in the literature are related to both COVID19-positive and COVID-19-negative patients, with only a small amount of them considering surgically treated femur fractures. According to Mamarelis et al., the global mortality rate of patients with both COVID-19-positive and COVID19-negative femur fractures surgically or conservatively treated was $22 \%$, increasing to $54.5 \%$ in positive patients. However, in surgically treated patients, the global mortality rate decreased to $12.9 \%$, which increased to $37.5 \%$ in positive patients. These data supported the hypothesis of Mamarelis et al. of higher mortality rate for COVID19-positive patients; however, no distinction in the type of treatment, the time before surgery, and the comorbidities of patients were reported [26]. Despite the time of surgery was not investigated in our study, the FRF group showed twice the incidence of death than the prosthesis replacement group. This difference could be related to the different types of fracture, where lateral fractures are more likely to undergo surgical intervention. Moreover, the number of patients who underwent FRF and prosthesis replacement was quite different (35 vs. 21). However, statistical analysis revealed no significant difference. The same higher mortality rate for intramedullary fixation was observed by Ekinci et al. [27], the study of Kim et al. [28], and the meta-analysis of Ma et al. [29]. Despite fracture types were different, all revealed a higher mortality rate for osteosynthesis in the first months from treatment, becoming similar after one or two years of follow-up, but no significant differences were found.

Our study has several limitations. Some are related to the nature of the study and study design; the lack of a control group, the retrospective nature of the study, and the lack of randomization are some of the major limitations. The sample size is also limited, despite the number of patients included in the study is the widest present in literature. Moreover, we do not know the exact mortality rate of conservatively treated COVID-19 femur fracture. This evaluation was not possible due to the impossibility of recruiting the patients. Finally, it was not possible to analyze 48-hour treatment cutoff; several reasons did not allow us to perform this analysis. First of all, before surgery, all patients were screened for COVID-19 infection with a nasopharyngeal swab. The first generation of swabs took about 24 hours before obtaining the results, and after, the positive patients were treated in a segregated COVID-19 theatre. With the new generation of swabs, we were able to obtain results earlier; however, they became available only after several months from the beginning of the first pandemic wave. This linear protocol was possible only in the hospitals reserved for the treatment of positive patients. Some hospitals were considered COVID19 free, with no segregated COVID-19 theatre, and in this case, patients were moved to designed COVID-19 hospital. After the arrival to a COVID-19 hospital, patients repeated another swab to confirm the positive result, with the delay of operation. Moreover, the access to a segregated COVID-19 theater is settled by rigorous protocols to preserve COVID19-free areas, which required time to be performed. Considering all these factors, the amount of time before access to the first emergency department and a secondary transfer to a designed COVID-19 hospital, or the compliance with strict protocols, surgery before 48 hour from trauma was rarely feasible.

\section{Conclusions}

A comparable 30-day mortality rate of patients with COVID-19 infection was observed compared with the literature of non-COVID-19 infection patients. Although the 60-day mortality raised and remained stable at the 90-day follow-up, more long-term follow-up is needed, since the 60-day mortality rate is higher than non-COVID-19-infected patients at 6 months compared to literature. Moreover, patients with pre-existing comorbidities and symptomatic COVID-19 infection requiring a high volume of O2 therapy have higher incidence of 90-day mortality when surgically treated.

Author contributions FF and GC equally participated in the design of the study, data collection, statistical analysis, article writing, and revision of the manuscript. AM, SR, AF, EE, KZ, FZ, and GB, participated in data collection, writing of the manuscript, and language editing. All authors read and approve the final version of the manuscript.

Data availability Data were not stored in a repository.

\section{Declarations}

Conflict of interest The authors declare no competing interests.

Ethical approval Local Ethical Committee approval was received.

Informed consent Written informed consent was obtained from all patients involved in the study. Consent for publication All patients gave their written informed consent for the publication of the study.

\section{References}

1. Brüssow H (2020) Immunology of COVID-19. Environ Microbiol 22:4895-4908. https://doi.org/10.1111/1462-2920.15302

2. Yüce M, Filiztekin E, Özkaya KG (2021) COVID-19 diagnosis-a review of current methods. Biosens Bioelectron. https://doi.org/ 10.1016/j.bios.2020.112752 
3. WHO Director-General's opening remarks at the media briefing on COVID-19 - March 11th 2020. https://www.who.int/directorgeneral/speeches/detail/who-director-general-s-opening-remar ks-at-the-media-briefing-on-covid-19---11-march-2020. Accessed Apr 252021

4. Grasselli G, Pesenti A, Cecconi M (2020) Critical care utilization for the COVID-19 outbreak in Lombardy, Italy: early experience and forecast during an emergency response. JAMA J Am Med Assoc 323:1545-1546

5. Abrahamsen B, Van Staa T, Ariely R, Olson M, Cooper C (2009) Excess mortality following hip fracture: a systematic epidemiological review. Osteoporos Int 20:1633-1650

6. Kanis JA, Oden A, Johnell O, De Laet C, Jonsson B, Oglesby AK (2003) The components of excess mortality after hip fracture. Bone 32:468-473. https://doi.org/10.1016/S8756-3282(03) 00061-9

7. Dyer SM, Crotty M, Fairhall N, Magaziner J, Beaupre LA, Cameron ID, Sherrington C (2016) A critical review of the long-term disability outcomes following hip fracture. BMC Geriatr 16:1-14

8. Matassi F, Carulli C, Munz G, Lualdi C, Civinini R, Innocenti M (2015) Preliminary results of an early vs delayed timing of surgery in the management of proximal femur fragility fractures. Clin Cases Miner Bone Metab 12:11-17

9. Civinini R, Paoli T, Cianferotti L, Cartei A, Boccaccini A, Peris A, Brandi ML, Rostagno C, Innocenti M (2019) Functional outcomes and mortality in geriatric and fragility hip fractures-results of an integrated, multidisciplinary model experienced by the "Florence hip fracture unit." Int Orthop 43:187-192. https://doi.org/10.1007/ s00264-018-4132-3

10. Frost SA, Nguyen ND, Center JR, Eisman JA, Nguyen TV (2013) Excess mortality attributable to hip-fracture: a relative survival analysis. Bone 56:23-29. https://doi.org/10.1016/j.bone.2013.05. 006

11. Rosso F, Dettoni F, Bonasia DE, Olivero F, Mattei L, Bruzzone M, Marmotti A, Rossi R (2016) Prognostic factors for mortality after hip fracture: operation within 48 hours is mandatory. Injury 47:S91-S97. https://doi.org/10.1016/j.injury.2016.07.055

12 Castronuovo E, Pezzotti P, Franzo A, Di Lallo D, Guasticchi G (2011) Early and late mortality in elderly patients after hip fracture: a cohort study using administrative health databases in the Lazio region, Italy. BMC Geriatr. https://doi.org/10.1186/ 1471-2318-11-37

13. Weekly epidemiological update on COVID-19 - April 20th 2021. https://www.who.int/publications/m/item/weekly-epidemiolo gical-update-on-covid-19---20-april-2021. Accessed Apr 252021

14. Hoffmann C, Wolf E (2021) Older age groups and countryspecific case fatality rates of COVID-19 in Europe, USA and Canada. Infection 49:111-116. https://doi.org/10.1007/ s15010-020-01538-w

15. Omori R, Matsuyama R, Nakata Y (2020) The age distribution of mortality from novel coronavirus disease (COVID-19) suggests no large difference of susceptibility by age. Sci Rep. https://doi. org/10.1038/s41598-020-73777-8

16. Onder G, Rezza G, Brusaferro S (2020) Case-fatality rate and characteristics of patients dying in relation to COVID-19 in Italy. JAMA J Am Med Assoc 323:1775-1776

17. Contou D, Cally R, Sarfati F, Desaint P, Fraissé M, Plantefève $G$ (2021) Causes and timing of death in critically ill COVID-19 patients. Crit Care. https://doi.org/10.1186/s13054-021-03492-x
18. Moran CG, Wenn RT, Sikand M, Taylor AM (2005) Early mortality after hip fracture: is delay before surgery important? J Bone Jt Surg Ser A 87:483-489. https://doi.org/10.2106/JBJS.D.01796

19. Simunovic N, Devereaux PJ, Sprague S, Guyatt GH, Schemitsch E, DeBeer J, Bhandari M (2010) Effect of early surgery after hip fracture on mortality and complications: systematic review and meta-analysis. CMAJ 182:1609-1616

20. Bokshan SL, Marcaccio SE, Blood TD, Hayda RA (2018) Factors influencing survival following hip fracture among octogenarians and nonagenarians in the United States. Injury 49:685-690. https://doi.org/10.1016/j.injury.2018.02.004

21. Brogan K, Akehurst H, Bond E, Gee C, Poole W, Shah NN, McChesney S, Nicol S (2016) Delay to surgery does not affect survival following osteoporotic femoral fractures. Injury 47:2294 2299. https://doi.org/10.1016/j.injury.2016.07.003

22. Schoeneberg C, Aigner R, Pass B, Volland R, Eschbach D, Peiris SE, Ruchholtz S, Lendemans S (2021) Effect of time-to-surgery on in-house mortality during orthogeriatric treatment following hip fracture: a retrospective analysis of prospectively collected data from 16,236 patients of the AltersTraumaRegister DGUß. Injury 52:554-561. https://doi.org/10.1016/j.injury.2020.09.007

23. Dallari D, Zagra L, Cimatti P, Guindani N, D’Apolito R, Bove F, Casiraghi A, Catani F, D'Angelo F, Franceschini M, Massè A, Momoli A, Mosconi M, Ravasi F, Rivera F, Zatti G, Castelli CC (2021) Early mortality in hip fracture patients admitted during first wave of the COVID-19 pandemic in Northern Italy: a multicentre study. J Orthop Traumatol. https://doi.org/10.1186/ s10195-021-00577-9

24. Hu F, Jiang C, Shen J, Tang P, Wang Y (2012) Preoperative predictors for mortality following hip fracture surgery: a systematic review and meta-analysis. Injury 43:676-685

25. CovidSurg Collaborative; GlobalSurg Collaborative (2021) Timing of surgery following SARS-CoV-2 infection: an international prospective cohort study. Anaesthesia 76:748-758. https://doi.org/ 10.1111/anae. 15458

26. Mamarelis G, Oduoza U, Chekuri R, Estfan R, Greer T (2020) Mortality in patients with proximal femoral fracture during the COVID-19 pandemic. JBJS Open Access 5(e20):00086. https:// doi.org/10.2106/jbjs.oa.20.00086

27. Ekinci Y, Gürbüz K, Batin S, Kahraman M, Dogar F, Erten ZK (2020) A multicenter intertrochanteric fracture study in the elderly: hemiarthroplasty versus proximal femoral nailing. Jt Dis Relat Surg 31:209-217. https://doi.org/10.5606/EHC.2020.72421

28. Kim JW, Shon HC, Song SH, Lee YK, Koo KH, Ha YC (2020) Reoperation rate, mortality and ambulatory ability after internal fixation versus hemiarthroplasty for unstable intertrochanteric fractures in elderly patients: a study on Korean Hip Fracture Registry. Arch Orthop Trauma Surg 140:1611-1618. https://doi.org/ 10.1007/s00402-020-03345-2

29. Ma HH, Chou TFA, Tsai SW, Chen CF, Wu PK, Chen WM (2019) Outcomes of internal fixation versus hemiarthroplasty for elderly patients with an undisplaced femoral neck fracture: a systematic review and meta-analysis. J Orthop Surg Res 14:320

Publisher's note Springer Nature remains neutral with regard to jurisdictional claims in published maps and institutional affiliations. 